\title{
FUNCTIONAL DIFFERENTIAL EQUATIONS WITH ITERATED DELAY - A SURVEY
}

\author{
Mihail M. Konstantinov \\ University of Architecture, Civil Engineering and Geodesy \\ 1046 Sofia, BULGARIA \\ e-mail: mmk_fte@uacg.bg
}

\begin{abstract}
The paper deals with a special class of neutral functional differential equations (FDE): the so called FDE with iterated delay. They are special equations of superneutral type. Equations with iterated delay had been introduced and studied by Prof. D.D. Bainov (1933-2011) and the author of this paper in the period 1972-1978. As a result a number of papers in this area had been published and the term "FDE with iterated delay" was accepted in the literature.
\end{abstract}

AMS Subject Classification: $34 \mathrm{~K} 05,34 \mathrm{~K} 10$

Key Words: differential functional equations, iterated delay, initial value problems, boundary value problems, periodic problems

Dedicated to the bright memory of my

friend and teacher Prof. Drumi Bainov

\section{Introduction and Notation}

FDE with complex dependence of the delay on the solution and its derivatives arise in many areas of science and engineering. To define a general vector functional differential equation with retarded argument we shall introduce some standard notations as follows, see also [4].

Let $\mathbb{R}=(-\infty,+\infty), \mathbb{R}_{+}=[0,+\infty), \mathbb{R}^{n \times m}$ be the space of $(n \times m)$-matrices over $\mathbb{R}, \mathbb{R}^{n}=\mathbb{R}^{n \times 1}$ and $\|\cdot\|: \mathbb{R}^{n} \rightarrow \mathbb{R}_{+}$be a norm in $\mathbb{R}^{n}$. For a closed

Received: August 1, 2016

Published: 1 септември 2016 г. (c) 2016 Academic Publications, Ltd.

url: www.acadpubl.eu 
interval $J \subset \mathbb{R}$ we denote by $C\left(J, \mathbb{R}^{n}\right)$ the Banach space of continuous functions $J \rightarrow \mathbb{R}^{n}$ with topology of uniform convergence. In particular, given any two numbers $r, a \in \mathbb{R}_{+}$we use the notation

$$
\mathrm{C}[-r, a]=C\left([-r, a], \mathbb{R}^{n}\right)
$$

for the Banach space of continuous functions $\varphi:[-r, a] \rightarrow \mathbb{R}^{n}$ with norm

$$
\|\varphi\|_{\mathrm{C}}=\max \{\|\varphi(s)\|: s \in[-r, a]\}
$$

For fixed $t \in[-r, a]$ and $\mathbf{x} \in \mathrm{C}[-r, a]$ we define $\mathbf{x}_{t} \in \mathrm{C}[-r, 0]$ by

$$
\mathbf{x}_{t}(s)=\mathbf{x}(t+s), s \in[-r, 0]
$$

Let finally $\mathrm{D}[-r, 0]$ be a subset of $\mathbb{R} \times \mathbf{C}[-r, 0]$ and $\mathbf{f}: \mathbf{D}[-r, 0] \rightarrow \mathbb{R}^{n}$ be a given function.

\section{Problem Statement}

\subsection{Functional Differential and Difference Equations}

A pretty general functional differential equation has the form

$$
\mathbf{x}^{\prime}(t)=\mathbf{f}\left(t, \mathbf{x}_{t}\right), t>0
$$

In turn, a functional difference equation may be written as

$$
\mathbf{x}(t)=\mathbf{f}\left(t, \mathbf{x}_{t}\right)
$$

Particular cases of the right hand side $\mathbf{f}\left(t, \mathbf{x}_{t}\right)$ are

$$
\begin{aligned}
\mathbf{f}\left(t, \mathbf{x}_{t}\right) & =\mathbf{F}(t, \mathbf{x}(t)) \\
\mathbf{f}\left(t, \mathbf{x}_{t}\right) & =\mathbf{F}\left(t, \mathbf{x}\left(h_{1}(t)\right), \mathbf{x}\left(h_{2}(t)\right), \ldots, \mathbf{x}\left(h_{p}(t)\right)\right) \\
\mathbf{f}\left(t, \mathbf{x}_{t}\right) & \left.=\mathbf{F}\left(t, \int_{-r}^{0} \mathbf{g}(t, s, \mathbf{x}(t+s)) \mathrm{d} s\right)\right) \\
t & -r \leq h_{j}(t) \leq t(j=1,2, \ldots, p)
\end{aligned}
$$

We may also define equations with more complex delays such as

$$
\mathbf{f}\left(t, \mathbf{x}_{t}\right)=\mathbf{F}\left(t, \mathbf{x}\left(h_{1}(t, \mathbf{x}(t))\right), \mathbf{x}\left(h_{2}(t, \mathbf{x}(t))\right), \ldots, \mathbf{x}\left(h_{p}(t, \mathbf{x}(t))\right)\right)
$$


An interesting class of functional differential and difference equations are equations with iterated delay, e.g.

$$
\mathbf{f}\left(t, \mathbf{x}_{t}\right)=\mathbf{F}\left(t, \mathbf{x}(t), \mathbf{x}\left(\tau_{0}\right)\right)
$$

where

$$
\begin{aligned}
\tau_{k}= & \tau_{k}\left(t, \mathbf{x}(t), \mathbf{x}\left(\tau_{k+1}\right)\right) \\
& (k=0,1, \ldots, m-1) \\
\tau_{m}= & \tau_{m}(t, \mathbf{x}(t))
\end{aligned}
$$

When dealing with such equations it is convenient to suppose that the solution is Lipschitz continuous. To guarantee that such a solution exists and/or that it is unique, we suppose that all functions are Lipschitz continuous in all their arguments and that the corresponding Lipschitz constants satisfy a set of restrictions.

For equations (1) and (2) we may define initial or boundary conditions. An initial condition is $\mathbf{x}_{0}=\varphi$, or, equivalently

$$
\mathbf{x}(t)=\varphi(t), t \in[-r, 0]
$$

where $\varphi \in \mathrm{C}[-r, 0]$ is a given initial function. The initial value problem (1), (3) may be written as an integral equation

$$
\begin{aligned}
\mathbf{x}_{0} & =\varphi \\
\mathbf{x}(t) & =\varphi(0)+\int_{0}^{t} \mathbf{f}\left(s, \mathbf{x}_{s}\right) \mathrm{d} s, t>0
\end{aligned}
$$

For a given $T>0$ a boundary condition may be defined as

$$
\mathbf{b}\left(\mathbf{x}_{T}\right)=\mathbf{0}
$$

where $\mathbf{b}: \mathrm{C}[0, T] \rightarrow \mathbb{R}^{n}$. In this case (4) is an equation for determining the vector $\varphi(0)$. Particular cases of (4) considered later on are the two-point

$$
\mathbf{A}_{0} \mathbf{x}(0)+\mathbf{A}_{1} \mathbf{x}(T)=\mathbf{0}
$$

and the multi-point

$$
\sum_{i=0}^{p} \mathbf{A}_{i} \mathbf{x}\left(t_{i}\right)=\mathbf{b}\left(\mathbf{x}\left(t_{0}\right), \mathbf{x}\left(t_{1}\right), \ldots, \mathbf{x}\left(t_{p}\right)\right)
$$

boundary conditions, where

$$
0=t_{0}<t_{1}<\cdots<t_{p}=T, \mathbf{A}_{0}, \mathbf{A}_{1}, \ldots, \mathbf{A}_{p} \in \mathbb{R}^{n \times n}
$$

and $\mathbf{b}:\left(\mathbb{R}^{n}\right)^{p+1} \rightarrow \mathbb{R}^{n}$. 


\subsection{Neutral Functional Differential Equations}

Neutral functional differential equations depend on past states of the derivative of the solution. An example of such equation is

$$
\mathbf{x}^{\prime}(t)=\mathbf{F}\left(t, \mathbf{x}(t), \mathbf{x}(\tau), \mathbf{x}^{\prime}(t), \mathbf{x}^{\prime}(\delta)\right)
$$

where

$$
\tau=\tau(t), \delta=\delta(t)
$$

The transformed arguments may depend also on the solution, e.g.

$$
\tau=\tau(t, \mathbf{x}(t)), \delta=\delta(t, \mathbf{x}(t))
$$

In a more involved statement the transformed arguments depend on the derivative as well,

$$
\tau=\tau\left(t, \mathbf{x}(t), \mathbf{x}^{\prime}(t)\right), \delta=\delta\left(t, \mathbf{x}(t), \mathbf{x}^{\prime}(t)\right)
$$

Relations (5) and (6) define a functional differential equation of superneutral type.

A rather general neutral functional differential equation may be written in the form [4]

$$
\frac{\mathrm{d}}{\mathrm{d} t} \mathbf{D}\left(t, \mathbf{x}_{t}\right)=\mathbf{f}\left(t, \mathbf{x}_{t}\right)
$$

where $\mathbf{D}, \mathbf{f} \in \mathbf{D}[-r, 0]$. For such equations we may define initial and boundary value problems as in Section 2.1. We also stress that functional difference equations are in fact particular cases of neutral functional differential equations.

Interesting case of superneutral functional differential equations are equations with iterated delay such as

$$
\mathbf{x}^{\prime}(t)=\mathbf{F}\left(t, \mathbf{x}(t), \mathbf{x}\left(\tau_{0}\right), \mathbf{x}^{\prime}(t), \mathbf{x}\left(\delta_{0}\right)\right)
$$

where

$$
\begin{aligned}
\tau_{k}= & \tau_{k}\left(t, \mathbf{x}(t), \mathbf{x}\left(\tau_{k+1}\right), \mathbf{x}^{\prime}(t), \mathbf{x}\left(\delta_{k+1}\right)\right) \\
\delta_{k}= & \delta_{k}\left(t, \mathbf{x}(t), \mathbf{x}\left(\tau_{k+1}\right), \mathbf{x}^{\prime}(t), \mathbf{x}\left(\delta_{k+1}\right)\right) \\
& (k=0,1, \ldots, m-1) \\
\tau_{m}= & \tau_{m}\left(t, \mathbf{x}(t), \mathbf{x}^{\prime}(t)\right), \delta_{m}=\delta_{m}\left(t, \mathbf{x}(t), \mathbf{x}^{\prime}(t)\right)
\end{aligned}
$$

In what follows a survey is given on published existence and uniqueness results for initial and boundary value problems for superneutral functional differential equations with iterated delay of the form (7), (8). The first papers devoted to such equations are $[8,9]$. 


\section{Initial Value Problems}

In this section we describe existence and uniqueness results concerning initial value problems (IVB) for functional differential and functional difference equations. With exception of Section 3.1 we consider vector equations.

\subsection{Scalar Equations with Extremes}

The first papers dealing with such equations had been [8, 9]. The initial value problem considered in [8] is scalar and contains 'maxima' as follows.

$$
\begin{gathered}
x^{\prime}(t)=f\left(t, x(t), \bar{x}(t), \widetilde{x}(t), x\left(\tau_{0}\right), x^{\prime}(t), \bar{x}^{\prime}(t), \widetilde{x}^{\prime}(t), x^{\prime}\left(\delta_{0}\right)\right), t>0 \\
x(t)=\varphi(t), x^{\prime}(t)=\varphi^{\prime}(t), t \leq 0
\end{gathered}
$$

where

$$
\bar{x}(t)=\sup \{x(u): 0 \leq u \leq t\}, \widetilde{x}(t)=\sup \{x(u): t-h \leq u \leq t\}
$$

and $h>0$ is a given constant.

The interesting part of the above problem are the iterated delays $\tau_{k}$ and $\delta_{k}$ defined by the recurrence relations

$$
\begin{aligned}
\tau_{k}= & \tau_{k}\left(t, x(t), \bar{x}(t), \widetilde{x}(t), x\left(\tau_{k+1}\right), x^{\prime}(t), \bar{x}^{\prime}(t), \widetilde{x}^{\prime}(t), x^{\prime}\left(\delta_{k+1}\right)\right) \\
\delta_{k}= & \delta_{k}\left(t, x(t), \bar{x}(t), \widetilde{x}(t), x\left(\tau_{k+1}\right), x^{\prime}(t), \bar{x}^{\prime}(t), \widetilde{x}^{\prime}(t), x^{\prime}\left(\delta_{k+1}\right)\right) \\
& (k=0,1, \ldots, m-1) \\
\tau_{m}= & \tau_{m}\left(t, x(t), \bar{x}(t), \widetilde{x}(t), x^{\prime}(t), \bar{x}^{\prime}(t), \widetilde{x}^{\prime}(t)\right) \\
\delta_{m}= & \left.\delta_{m}\left(t, x(t), \bar{x}(t), \widetilde{x}(t), x^{\prime}(t), \bar{x}^{\prime}(t), \widetilde{x}^{\prime}(t)\right)\right)
\end{aligned}
$$

for some integer $m \geq 1$.

For simplicity, consider the above class of FDE without maxima, namely

$$
\begin{gathered}
x^{\prime}(t)=f\left(t, x(t), x\left(\tau_{0}\right), x^{\prime}(t), x^{\prime}\left(\delta_{0}\right)\right), t>0 \\
x(t)=\varphi(t), x^{\prime}(t)=\varphi^{\prime}(t), t \leq 0
\end{gathered}
$$

and

$$
\begin{aligned}
\tau_{k}= & \tau_{k}\left(t, x(t), x\left(\tau_{k+1}\right), x^{\prime}(t), x^{\prime}\left(\delta_{k+1}\right)\right) \\
\delta_{k}= & \delta_{k}\left(t, x(t), x\left(\tau_{k+1}\right), x^{\prime}(t), x^{\prime}\left(\delta_{k+1}\right)\right) \\
& (k=0,1, \ldots, m-1)
\end{aligned}
$$




$$
\begin{aligned}
& \tau_{m}=\tau_{m}\left(t, x(t), x^{\prime}(t)\right) \\
& \delta_{m}=\delta_{m}\left(t, x(t), x^{\prime}(t)\right)
\end{aligned}
$$

It is supposed that the functions involved in the above relations are continuous in $t$ and Lipschitz continuous in all other arguments.

Three theorems have been proven in [8] for the system (11), (12): for existence of at least one solution on a finite interval $[0, T]$, for existence of unique solution on another finite interval $\left[0, T^{*}\right]$, and for existence of unique solution on the infinite interval $[0, \infty)$. In all cases the derivative of the solution is Lipschitz continuous. The first and second theorems are proved using the Schauder and Banach fixed point principles, respectively. The third theorem is proved by a recurrent process of constructing the solution on a system of finite intervals whose union is the whole semi-axis $[0, \infty)$.

Another type of extremal systems governed by FDE with iterated delay has been considered in [9], namely

$$
\begin{aligned}
x^{\prime}(t) & =\min _{q \in \mathbb{R}} \max _{p \in \mathbb{R}} f\left(p, q, t, x(t), x\left(\tau_{0}\right), x^{\prime}(t), x^{\prime}\left(\delta_{0}\right)\right), t>0 \\
x(t) & =\varphi(t), x^{\prime}(t)=\varphi^{\prime}(t), t \leq 0
\end{aligned}
$$

where

$$
\begin{aligned}
\tau_{k}= & \tau_{k}\left(t, x\left(\tau_{k+1}, x^{\prime}(t), x^{\prime}\left(\delta_{k+1}\right)\right)\right. \\
\delta_{k}= & \delta_{k}\left(t, x\left(\tau_{k+1}, x^{\prime}(t), x^{\prime}\left(\delta_{k+1}\right)\right)\right. \\
& (k=0,1, \ldots, m-1) \\
\tau_{m}= & \tau_{m}\left(t, x(t), x^{\prime}(t)\right) \\
\delta_{m}= & \delta_{m}\left(t, x(t), x^{\prime}(t)\right)
\end{aligned}
$$

\subsection{Functional Difference Equations}

An initial value problem for a class of functional-difference equations is considered in [6]. It is of the form

$$
\begin{aligned}
& \mathbf{x}(t)=\mathbf{f}\left(t, \mathbf{x}(t), \mathbf{x}\left(\tau_{0}\right)\right), t>0 \\
& \mathbf{x}(t)=\varphi(t), t<0
\end{aligned}
$$

where $\mathbf{x}(t) \in \mathbb{R}^{n}$ and $\mathbf{f}: \mathbb{R} \times\left(\mathbb{R}^{n}\right)^{2}$. The delay $\tau_{0}$ is defined by

$$
\begin{aligned}
\tau_{k} & =\tau_{k}\left(t, \mathbf{x}(t), \mathbf{x}\left(\tau_{k+1}\right)\right),(k=0,1, \ldots, m-1) \\
\tau_{m} & =\tau_{m}(t, \mathbf{x}(t))
\end{aligned}
$$

Theorems for existence and for existence and uniqueness of the solutions are proved. 


\subsection{Functional Differential Equations}

In [13] a general type initial value problem for vector differential-functional equations of superneutral type with iterated delay is considered, namely

$$
\mathbf{y}(t)= \begin{cases}\mathbf{f}\left(t, \mathbf{x}_{t}, \mathbf{x}\left(\tau_{0}\right), \mathbf{y}(t), \mathbf{y}\left(\delta_{0}\right)\right) & t>0 \\ \psi(t) & t \leq 0\end{cases}
$$

where $\mathbf{y}(t) \in \mathbb{E}^{n}(\mathbb{E}$ is a numeral field) and

$$
\mathbf{x}(t)=\xi_{0}+\int_{0}^{t} \mathbf{y}(s) \mathrm{d} s
$$

Here $\mathbf{x}_{t}$ is a restriction of the function $\mathbf{x}$ on the interval $(-\infty, t]$. The arguments $\tau_{0}$ and $\delta_{0}$ are defined via the recurrent relations

$$
\begin{aligned}
\tau_{k}= & \tau_{k}\left(t, \mathbf{x}_{t}, \mathbf{x}\left(\tau_{k+1}\right), \mathbf{y}(t), \mathbf{y}\left(\delta_{k+1}\right)\right) \\
\delta_{k}= & \delta_{k}\left(t, \mathbf{x}_{t}, \mathbf{x}\left(\tau_{k+1}\right), \mathbf{y}(t), \mathbf{y}\left(\delta_{k+1}\right)\right) \\
& (k=0,1, \ldots, m-1) \\
\tau_{m}= & \tau_{m}\left(t, \mathbf{x}_{t}, \mathbf{y}(t)\right) \\
\delta_{m}= & \delta_{m}\left(t, \mathbf{x}_{t}, \mathbf{y}(t)\right)
\end{aligned}
$$

Two theorems are stated: for existence and for existence and uniqueness of the solution. Full proofs of these results is published in [14].

Other initial value problems for superneutral functional differential equations are stutdied in $[12,16]$.

\subsection{Functional Integro-Differential Equations}

Functional integro-differential equations with iterated delay had been considered for the first time in [21]. The problem under consideration is

$$
\begin{aligned}
\mathbf{x}^{\prime}(t) & =\mathbf{f}\left(t, \mathbf{x}(t), \mathbf{x}\left(\delta_{0}\right), \mathbf{x}\left(\tau_{0}\right), \mathbf{x}^{\prime}(t), \mathbf{x}^{\prime}\left(\delta_{0}\right), \mathbf{W}\left[t, \mathbf{x}, \mathbf{x}^{\prime}\right]\right), t>c \\
\mathbf{x}(t) & =\varphi(t), \mathbf{x}^{\prime}(t)=\varphi^{\prime}(t), t \leq c
\end{aligned}
$$

where

$$
\mathbf{W}\left[t, \mathbf{x}, \mathbf{x}^{\prime}\right]=\int_{c}^{t} \mathbf{F}\left(t, s, \mathbf{x}(s), \mathbf{x}\left(\delta_{0}\right), \mathbf{x}\left(\tau_{0}\right), \mathbf{x}^{\prime}(s), \mathbf{x}^{\prime}\left(\delta_{0}\right)\right) \mathrm{d} s
$$

and

$$
\tau_{k}=\tau_{k}\left(t, \mathbf{x}(t), \mathbf{x}\left(\delta_{k+1}\right), \mathbf{x}\left(\tau_{k+1}\right), \mathbf{x}^{\prime}(t), \mathbf{x}^{\prime}\left(\delta_{k+1}\right)\right)
$$




$$
\begin{aligned}
\delta_{k}= & \delta_{k}\left(t, \mathbf{x}(t), \mathbf{x}\left(\delta_{k+1}\right), \mathbf{x}\left(\tau_{k+1}\right), \mathbf{x}^{\prime}(t), \mathbf{x}^{\prime}\left(\delta_{k+1}\right)\right) \\
& (k=0,1, \ldots, m) \\
\tau_{m+1}= & \delta_{m+1}=t
\end{aligned}
$$

Three theorems for existence and uniqueness of the solution are proved and an example is considered. The norm of Bielecki [2] has been used, see also [3, 20].

\section{Boundary Value Problems}

The first paper dealing with boundary value problems for FDE of superneutral type is [5]. Here the differential equation

$$
\mathbf{x}^{\prime}(t)=\mathbf{f}\left(t, \mathbf{x}(t), \mathbf{x}^{\prime}(t), \mathbf{x}\left(\tau_{0}\right), \mathbf{x}^{\prime}\left(\tau_{0}\right)\right), t \in[0, T]
$$

is subject to the two-point boundary condition

$$
\mathbf{A x}(0)+\mathbf{B} \mathbf{x}(T)=\mathbf{0}
$$

where $\mathbf{x}(t) \in \mathbb{R}^{n}, \mathbf{f}:[0, T] \times\left(\mathbb{R}^{n}\right)^{4} \rightarrow \mathbb{R}^{n}$ and

$$
\begin{aligned}
\tau_{k}= & \tau_{k}\left(t, \mathbf{x}(t), \mathbf{x}^{\prime}(t), \mathbf{x}\left(\tau_{k+1}\right), \mathbf{x}^{\prime}\left(\tau_{k+1}\right)\right) \\
& (k=0,1, \ldots, m-1) \\
\tau_{m}= & \tau_{m}\left(t, \mathbf{x}(t), \mathbf{x}^{\prime}(t)\right)
\end{aligned}
$$

It is supposed that the matrix $\mathbf{A}+\mathbf{B}$ is invertible. Three theorems are proved. In the first one sufficient conditions for existence of the solution are given without restrictions on $T$. In the next two theorems existence and uniqueness of the solution studied. An illustrative example is also considered.

Another type of BVP is considered in [11]:

$$
\begin{aligned}
& \mathbf{x}^{\prime \prime}(t)=\mathbf{f}\left(t, \mathbf{x}(t), \mathbf{x}^{\prime}(t), \mathbf{x}^{\prime \prime}(t), \mathbf{x}\left(\tau_{0}\right), \mathbf{x}^{\prime}\left(\tau_{0}\right), \mathbf{x}^{\prime \prime}\left(\tau_{0}\right)\right) \\
& \mathbf{A}_{0} \mathbf{x}(0)+\mathbf{A}_{1} \mathbf{x}(T)+\mathbf{B}_{0} \mathbf{x}(0)+\mathbf{B}_{1} \mathbf{x}_{1}(T)=\mathbf{0} \\
& \mathbf{C}_{0} \mathbf{x}(0)+\mathbf{C}_{1} \mathbf{x}(T)+\mathbf{D}_{0} \mathbf{x}(0)+\mathbf{D}_{1} \mathbf{x}_{1}(T)=\mathbf{0}
\end{aligned}
$$

where $\mathbf{x}(t) \in \mathbb{R}^{n}, \mathbf{f}:[0, T] \times\left(\mathbb{R}^{n}\right)^{6} \rightarrow \mathbb{R}^{n}$ and

$$
\begin{aligned}
\tau_{k}= & \tau_{k}\left(t, \mathbf{x}(t), \mathbf{x}^{\prime}(t), \mathbf{x}^{\prime \prime}(t), \mathbf{x}\left(\tau_{k+1}\right), \mathbf{x}^{\prime}\left(\tau_{k+1}\right), \mathbf{x}^{\prime \prime}\left(\tau_{k+1}\right)\right) \\
& (k=0,1, \ldots, m-1) \\
\tau_{m}= & \tau_{m}\left(\mathbf{x}(t), \mathbf{x}^{\prime}(t), \mathbf{x}^{\prime \prime}(t)\right)
\end{aligned}
$$


Two theorems are given: for existence and for existence and uniqueness of the solution.

A multi-point nonlinear BVP is considered in [15] for the vector differential equation

$$
\mathbf{x}^{\prime}(t)=\mathbf{f}\left(t, \mathbf{x}(t), \mathbf{x}^{\prime}(t), \mathbf{x}\left(\tau_{0}\right), \mathbf{x}^{\prime}\left(\tau_{0}\right)\right), t \in\left[t_{1}, t_{2}\right]
$$

with nonlinear boundary condition

$$
\sum_{i=1}^{N} \mathbf{A}_{i} \mathbf{x}\left(t_{i}\right)=\Gamma\left(\mathbf{x}\left(t_{1}\right), \mathbf{x}\left(t_{2}\right), \ldots, \mathbf{x}\left(t_{N}\right)\right)
$$

where

$$
\begin{aligned}
\tau_{k}= & \tau_{k}\left(t, \mathbf{x}(t), \mathbf{x}^{\prime}(t), \mathbf{x}\left(\tau_{k+1}\right), \mathbf{x}^{\prime}\left(\tau_{k+1}\right)\right) \\
& (k=0,1, \ldots, m-1) \\
\tau_{m}= & \tau_{m}\left(t, \mathbf{x}(t), \mathbf{x}^{\prime}(t)\right)
\end{aligned}
$$

Theorems for existence and for existence and uniqueness are proved. It is stressed that the conditions for existence of a unique solution can slightly be weakened by using an appropriate Bielecki's norm [2].

Boundary value problems for superneutral functional differential equations are also considered in $[1,17,18,19]$.

\section{Periodic Solutions}

Paper [10] deals with periodic solutions of functional differential equations

$$
\mathbf{x}^{\prime \prime}(t)+\mathbf{\Omega}^{2} \mathbf{x}(t)=\mathbf{f}\left(t, \mathbf{x}(t), \mathbf{x}^{\prime}(t), \mathbf{x}^{\prime \prime}(t), \mathbf{x}\left(\tau_{0}\right), \mathbf{x}^{\prime}\left(\tau_{0}\right), \mathbf{x}^{\prime \prime}\left(\tau_{0}\right)\right)
$$

where $\mathbf{x}(t) \in \mathbb{R}^{n}, \mathbf{f}:[0, T] \times\left(\mathbb{R}^{n}\right)^{6} \rightarrow \mathbb{R}^{n}$, the function $\mathbf{f}$ is $T$-periodic in $t$, and

$$
\begin{aligned}
\tau_{k}= & \tau_{k}\left(t, \mathbf{x}(t), \mathbf{x}^{\prime}(t), \mathbf{x}\left(\tau_{k+1}\right), \mathbf{x}^{\prime}\left(\tau_{k+1}\right)\right) \\
& (k=0,1, \ldots, m-1) \\
\tau_{m}= & \tau_{m}\left(\mathbf{x}(t), \mathbf{x}^{\prime}(t)\right)
\end{aligned}
$$

Conditions for existence and uniqueness of the periodic solution are established and element-wise estimates for the solution and its derivatives are found. The results are formulated in five theorems. An example is considered. 


\section{References}

[1] D. Bainov, M. Konstantinov. On a boundary value problem with a generalized boundary condition for systems of superneutral differential equations. Differential Equations. Colloquia Math. Soc. J. Bolyai 15 (1975), pp. 51-61. North-Holland, Amsterdam, 1977 (ZMATH 355.34056, MR 0492708, РжМат 4Б363/79)

[2] A. Bielecki. Une remarque sur la méthode de Banach-Cacciopoli-Tikhonov dans la théorie des équations differentielles ordinaires. Bull. Acad. Polon. Sci. Sér. Math. Phys. Astr. 4 (1956), pp. 261-264

[3] C. Corduneanu. Bielecki's method in the theory of integral equations. Ann. Univ. Marie Curie-Sklodowska, Sér. A 38 (1984), no. 2, pp. 23-40

[4] J. Hale. Theory of Functional Differential Equations. Springer-Verlag, NY, 1977, ISBN 978-1-4612-9892-2

[5] M. Konstantinov. Existence and uniqueness of the solutions of boundary value problems for differential equations of superneutral type (in Russian). Math. Balkanica 3.32 (1973), pp. 213-219, ISSN 0205-3217 (ZMATH 299.34093, MR 0374597, РжМат 12Б162/74)

[6] M. Konstantinov. Existence and uniqueness of the solutions of a class of functionaldifference equations. Bull. Inst. Politehn. Iasi (N.S.) 20 (24) (1974), fasc. 3-4, sect. I, pp. 53-56, ISSN 1223-8139 (ZMATH 332.39006, MR 0385363, РжМат 2Б8/76)

[7] M. Konstantinov. On a two-point boundary value problem for an ordinary differential equation of superneutral type (in Russian). Rev. Anal. Numer. Theor. Approx. Math. 16 (39) (1974), 281-285 (ZMATH 397.34090, MR 0508789)

[8] M. Konstantinov, D. Bainov. Theorems of existence and uniqueness of the solution of certain differential equations of superneutral type (in Russian). Publ. Inst. Math. (N.S.), 14 (28) (1972), pp. 75-82, ISSN 0350-1302 (ZMATH 269.34054, MR 0387768, РжМат 12Б340/73)

[9] M. Konstantinov, D. Bainov. Existence and uniqueness of the solution of certain extremal-differential systems of superneutral type (in Russian). Arch. Math. 8 (28) (1972), no. 4, pp. 207-212, ISSN 0044-8753 (ZMATH 284.34070, MR 0330695, РжМат 4Б280/74)

[10] M. Konstantinov, D. Bainov. Existence and uniqueness of periodic solutions of a class of differential-functional equations of superneutral type (in Russian). Math. Balkanica 3.33 (1973), pp. 220-228, ISSN 0205-3217 (ZMATH 293.34103, MR 0374598, РжМат 12Б163/74)

[11] M. Konstantinov, D. Bainov. On a boundary value problem of second order for a system of differential equations of superneutral type (in Russian). Proc. Georgia Acad. Sci. 74 (1974), pp. 285-288, ISSN 0132-1447 (ZMATH 294.34049, MR 0364812, РжМат 12Б164/74)

[12] M. Konstantinov, D. Bainov. Existence and uniqueness of solutions of systems of differential equations of superneutral type (in Russian). Rev. Roum. Math. Pures Appl. 19 (1974), pp. 1221-1230, ISSN 0035-3965 (ZMATH 294.34048, MR 0361352, РжМат $6 \mathrm{5} 413 / 75)$

[13] M. Konstantinov, D. Bainov. On the existence of solutions of systems of differential equations of superneutral type with iterated delay (in Russian). Doklady AN USSR 217 (1974), pp. 519-521, ISSN 0869-5652, English translation: Soviet Math. Dokl. 15 (1974), pp. 1087-1089 (ZMATH 298.34063, MR 0364808, РжМат 12Б161/74) 
[14] M. Konstantinov, D. Bainov. The existence of the solutions of systems of functional equations of superneutral type with iterated delay (in Russian). Differenc. Uravn. 10 (1974), pp. 1988-1992, ISSN 0374-0641, English translation: Differential Equations 10 (1974), pp. 1532-1536 (ZMATH 303.34053, MR 0397112, РжМат 4Б314/75)

[15] M. Konstantinov, D. Bainov. Multi-point boundary value problem for nonlinear differential equations with time-lag, not solved for the derivative (in Russian). Differenc. Uravn. 11 (1975), pp. 1201-1205, ISSN 0374-0641, English translation: Differential Equations 11 (1975), pp. 902-905 (ZMATH 305.34099, 337.34066, MR 0374606, РжМат 11Б325/75)

[16] M. Konstantinov, D. Bainov. Theorems for existence and uniqueness of the solution for systems of differential equations of superneutral type with iterated delay (in Russian). Bull. Math. Soc. Sci. Math. Roum. 20 (68) (1976), pp. 151-158, ISSN 1220-3874 (ZMATH 364.34033, MR 0477374, РжМат 6Б221/78)

[17] M. Konstantinov, D. Bainov. On a boundary value problem with generalized boundary condition for systems of differential equations of superneutral type. Bul. Acad. Serbe Sci. Math. Natur. 55 (1976), no. 9, pp. 39-47, ISSN 0561-7332 (ZMATH 361.34060, MR 0430464)

[18] M. Konstantinov, D. Bainov. Multi-point boundary value problem for differential equations of superneutral type (in Russian). Studia Sci. Math. Hungarica 12 (1977), pp. 95-99, ISSN 0081-6906 (ZMATH 443.34016, MR 0568468, РжМат 10Б316/80)

[19] M. Konstantinov, D. Bainov. On a boundary value problem with generalized boundary condition for systems of differential equations of superneutral type (in Russian) Rev. Roum. Math. Pures Appl. 22 (1977), pp. 1241-1248, ISSN 0035-3965 (ZMATH 376.34054, MR 0508788, РжМат 6Б222/78)

[20] M. Kwapisz. An extension of Bielecki's method of proving of global existence and uniqueness results for functional equations. Ann. Univ. Marie Curie-Sklodowska, Sér. A 38 (1984), no. 2, pp. 59-68

[21] T. Nikolova, M. Konstantinov, D. Bainov. Theorems for existence and uniqueness of the solutions of integro-differential equations of superneutral type with iterated delay (in Russian). Math. Balkanica $\mathbf{3 . 4 7}$ (1973), pp. 388-396, ISSN 0205-3217 (ZMATH 288.34071, MR 0374600, РжМат 10Б385/74) 
